LA-ÜR-96- 863

TITLE:

AUTHOR(S):

SUBMITTED TO:

a.

MASTER

TIED TO:

8th ICFA Advanced Beam Dynamics Workshop

on Space Charged Dominated Beams and Applications

of High Brightness Beams

Bloomington, Indiana, October 11-13, 1995
REC $1 \mathrm{CHO}$

MAY 051905

OSTI

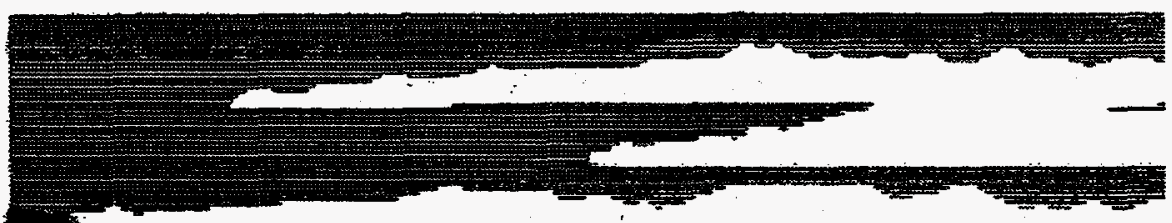

Los Alamos National Laboratony, an affirmative action/equal opportunity employer, is operated by the University of California for the U.S. Department of Energy under contract W-7405-ENG-36. By acceptance of this article, the publisher recognizes that the U.S. Government retains a nonexclusive, royalty-free license to publish or reproduce the published form of this contribution, or to allow others to do so, for U.S. Govemment purposes. The Los Alamos National Laboratory requests that the publisher identify this article as work performed under the auspices of the U.S. Department of Energy. 


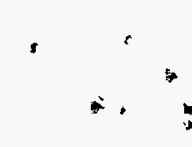

, 


\section{DISCLAIMER}

Portions of this document may be illegible in electronic image products. Images are produced from the best available original document. 


\title{
Emittance Concept and Growth Mechanisms
}

\author{
Thomas P. Wangler \\ Accelerator Operations and Technology Division \\ Los Alamos National Laboratory \\ Los Alamos, New Mexico 87545
}

\begin{abstract}
We present an introduction to the subjects of emittance and space-charge effects in charged-particle beams. This is followed by a discussion of three important topies that are at the frontier of this field. The first is a simple model, describing space-charge-induced emittance growth, which yields scaling formulas and some physical explanations for some of the surprising results. The second is a discussion of beam halo, an introduction to the particle-core model, and a brief summary of its results. The third topic is an introduction to the hypothesis of equipartitioning for collisionless particle beams.
\end{abstract}

\section{EMITTANCE CONCEPTS AND DEFINITIONS}

Beams are composed of collections of particles, which can be described by six normalized phase-space coordinates $\left(x, p_{x} / m c\right.$, $\left.y, p_{y} / m c, z, p_{z} / m c\right)$, where in If linacs, if $z$ is the direction of the center of momentum of the beam, $z$ and $p_{z} / m c$ are usually defined relative to a synchronous or reference particle. In general we can talk about a 6-D phase space distribution that describes the beam. As the beam is transported and accelerated, this distribution obeys Liouville's theorem, provided that the proper conditions are satisfied [1]. These are that 1) the continuity equation is valid in 6-D phase-space, which means that no particles are created or lost, and 2) the forces are derivable from a potential function that depends only on the three spatial coordinates and time. The second condition implies that we are ignoring the individual binary particle collisions, and representing the sum of the interparticle forces as a smoothed Coulomb force, averaged over all the particles. In most circumstances these conditions are a very good approximation, and the smoothed Coulomb force is commonly known as the space-charge force. Subject to these 
requirements the theorem states that the density in 6-D phase space, measured along the trajectory of a particle, is invariant, or equivalently that the 6-D volume enclosed by any isodensity contour is invariant.

Beam emittances are defined as a measure of the projections of the 6-D phase-space volume on the 2-D areas $x-p_{x}, y-p_{y}$, and $z-$ $\mathrm{p}_{\mathrm{z}}$ [2]. In linear focusing systems these areas are invariant, although their shapes will generally change as the beam propagates. Because most focusing systems are linear for small displacements, the invariance or approximate invariance of the emittances is the underlying reason why emittances are such useful quantities.

An arbitrary phase-space distribution can be described by an rms ellipse. The second moments of the distribution are used to define the ellipse parameters and the rms emittances. For example the definition of $\mathrm{rms}$ emittance in the $\mathrm{x}-\mathrm{p}_{\mathrm{x}}$ plane can be given as

$$
\varepsilon_{\mathrm{n}} \equiv \sqrt{\overline{\mathrm{x}^{2} \bar{p}_{x}^{2}}-{\overline{x p_{x}}}^{2}} / \mathrm{mc} .
$$

Similar results apply for the $y-p_{y}$ and $\left(z-z_{c}\right)-\left(p_{z}-p_{z c}\right)$ planes, where $z_{c}$ and $p_{z c}$ are the coordinates of the center of momentum of the beam. For historical reasons the emittance, defined by Eq.(1) is generally called the normalized emittance to distinguish it from the unnormalized emittance, which is specified in a phase space defined by position $x$ and divergence angle $x{ }^{\prime}=p_{x} / p_{z}$. In the paraxial approximation the unnormalized and normalized transverse emittances, respectively, are

$$
\begin{gathered}
\varepsilon=\sqrt{\overline{\mathrm{x}^{2} \mathrm{x}^{\prime 2}}-\overline{\mathrm{xx^{ \prime }}}}, \\
\varepsilon_{\mathrm{n}}=\varepsilon \beta \gamma=\beta \gamma \sqrt{\overline{\mathrm{x}^{2} \mathrm{x}^{\prime 2}}-\overline{\mathrm{xx}^{\prime}}},
\end{gathered}
$$


where $\beta$ is the ratio of the beam velocity over the speed of light, and $\gamma=1 / \sqrt{1-\beta^{2}}$ is the usual relativistic mass factor.

Any discussion of emittances should be accompanied by a word of caution to the reader, because the emittance conventions are not unique. The above definition of $\mathrm{rms}$ emittance corresponds to the convention introduced by Sacherer [3]: Another commonly used convention for rms emittance, introduced by Lapostolle [4], contains a factor of 4 in front of the square root of the above expressions. The situation is further confused by the fact that some Laboratories introduce other factors than the 4. Another confusion has to do with a factor of $\pi$, which is often appended, again for historical reasons. When a $\pi$ is attached to the numerical value, $\varepsilon$ becomes the area of an equivalent ellipse. For consistency we prefer not to introduce a factor of $\pi$, because in the envelope equation, to be introduced shortly, the symbol $\varepsilon$ generally represents an area divided by $\pi$ rather than an area. Until there is a consensus in the accelerator community about a convention for emittance, this author prefers to use the definitions of rms emittance, given by Eqs.(2) and (3), which have no additional factors of $\pi$ or 4 or any other numerical factors.

The rms emittance is invariant, if only linear forces act on the beam. Nonlinear forces produce filamentation with accompanying change in the rms emittance. When Liouville's theorem is satisfied, and when there is no coupling between the three orthogonal directions of motion, the true phase-space areas are preserved, even with nonlinear forces. However, the filamentation results in the capture of empty phase space between beam filaments [5], and intuitively, an effective emittance of the beam, including the area of both the filaments and the captured space, increases. For this case, it is found that the rms emittance also increases, essentially taking this emittance dilution into account. Therefore, the rms emittance is generally regarded as an effective emittance. It is interesting that under some conditions with nonlinear forces, the rms emittance may decrease [6]. Finally, the evolution of the rms beam size depends on the rms emittance, through an envelope 
equation. For transverse motion of continuous beams with elliptical symmetry, but otherwise arbitrary particle distributions, the rms envelope equations $[3,4]$, also known as the $K-V$ envelope equations [7], are

$$
\begin{aligned}
& \frac{d^{2} a_{x}}{d s^{2}}+k_{x}(s) a_{x}-\frac{\varepsilon_{x}^{2}}{a_{x}^{3}}-\frac{k}{2\left(a_{x}+a_{y}\right)}=0, \\
& \frac{d^{2} a_{y}}{d s^{2}}+k_{y}(s) a_{y}-\frac{\varepsilon_{y}^{2}}{a_{y}^{3}}-\frac{k}{2\left(a_{x}+a_{y}\right)}=0,
\end{aligned}
$$

where $a x$ and ay are the rms projections on the $x$ and $y$ axes, $k x$ and ky determine the strength of the focusing, $\varepsilon x$ and $\varepsilon y$ are unnormalized rms emittances, and $K$ is called the generalized perveance, given in terms of the particle charge $q$, mass $m$, and the beam current I, by

$$
K=\frac{q I}{2 \pi \varepsilon_{0} \gamma^{3} \beta^{3} m c^{3}}
$$

For a round beam in an ideal uniform focusing channel, the rms envelope equations become

$$
\frac{d^{2} a}{d s^{2}}+k_{0}^{2} a-\frac{\varepsilon^{2}}{a^{3}}-\frac{K}{4 a}=0,
$$

where $a$ is the rms projection on either the $x$ or $y$ axis, and $k 0$ is the transverse phase advance per unit length of the harmonic motion of a single particle without the space-charge force. The uniform channel is frequently used to represent a smooth approximation to the particle motion in a quadrupole focusing channel. The three force terms in Eqs.(3) and (5) represent the external focusing force, an outward pressure force associated with the emittance, and the space-charge defocusing force. 


\section{BEAMS WITH SPACE CHARGE}

Understanding space-charge- dominated beam is generally important to isolate the effects of space-charge, and to define the limits of high-intensity performance. The ratio of the space-charge to the emittance terms in the envelope equations can be used to determine when space-charge effects will become important. We refer to emittance dominated and space-charge dominated beams, according to

$$
\begin{aligned}
& \frac{\mathrm{Ka}^{2}}{4 \varepsilon^{2}} \ll 1, \text { emittance dominated, } \\
& \frac{\mathrm{Ka}^{2}}{4 \varepsilon^{2}} \gg 1 \text {, space-ch arge dominated. }
\end{aligned}
$$

A space-charge-dominated beam may be compared with a cold plasma, where collective effects are more important, whereas an emittance- dominated beam is associated primarily with random or thermal effects. Many accelerators that are characterized as high-current machines are not necessarily space-charge dominated, and are often associated with an intermediate regime, where $\mathrm{Ka}^{2} / 4 \varepsilon^{2}=1$. Because the external focusing force plays the same role as a background charge of opposite sign in a neutral plasma, it is not surprising that the behavior of beams with space-charge can sometimes be described in terms of concepts familiar from plasma physics. For example, a mismatched beam in a uniform channel will oscillate at the beam-plasma frequency, which can be written for an equivalent uniform-density beam as $\omega_{p}=\left(K \beta^{2} c^{2} / 2 a^{2}\right)^{1 / 2}$. If an effective thermal energy is defined as $k_{B} T=m c^{2} \beta^{2} \varepsilon^{2} / a^{2}$, a Debye length can be written as $\left.\lambda_{D}=R \varepsilon^{2} / K\right)^{1 / 2}$, which characterizes shielding effects in real beams; thus the particles tend to redistribute to shield the interior from the external fields. Also, at the edges of the beam, the particle density exhibits a characteristic Debye-length fall off. 
It is convenient to separate space-charge effects into two main categories, linear and nonlinear-force effects. The linear spacecharge fields produce defocusing, resulting in increased beam size, but with no emittance growth. The nonlinear fields also produce rms-emittance growth, and can be associated with significant halo formation at the periphery of the beam. Four categories of space-charge induced emittance growth can be identified [8], 1) charge redistribution [6,9], 2) kinetic-energy transfer $[10,11,12]$, rms mismatch $[13,14]$, and 4) structure resonances $[15,16,17]$. Although not all of these processes are fully understood, certain general remarks can be made. Charge redistribution describes the process in which the phase-space distribution rearranges to provide shielding of the interior of the beam from external fields. It can occur for rms-matched beams, and develops within a very short time scale; the full emittance growth typically occurs within only one quarter of a beam-plasma period. The emittance growth is represented quantitatively by a nonlinear field-energy theory in which the excess field energy associated with the nonuniform initial distribution is transferred to thermal energy. Kinetic-energy transfer describes the tendency of highly space-charge dominate beams to reduce, by means of the space-charge interactions, any large thermal asymmetries between the different degrees of freedom. Rms mismatch refers to emittance growth associated with rms mismatched beams, in which the excess energy of the mismatched beam oscillations is converted into thermal energy. Understanding this mechanism is important for control of large-amplitude beam halos. The structure resonance is a parametric resonance, in which the particle amplitudes are driven by interaction with the periodic-focusing structure. The most serious case is known as the envelope resonance or envelope instability, which leads to growth of the transverse emittances and beam envelopes. The envelope instability can be avoided by limiting the zerocurrent phase advance per focusing period to $\sigma_{0}<90^{\circ}$. The LBL beam-transport experiment [18] provides the best experimental study in a quadrupole channel on emittance growth space-charge dominated beams in alternating focusing channels. The other significant experiment is the University of Maryland experiment in a periodic solenoid channel, which is 
reported in many published papers, most of which can be found in Reference [19].

Much of what has been learned about space-charge-induced emittance growth has been the result of computer-simulation studies. Although analytic approaches have been limited by the complex nature of the problem, more analytic work is necessary to provide better physical understanding. A simple analytic model of emittance-growth from charge redistribution is an example of what is needed for improving our understanding of the scaling rules for emittance growth.

In this model [20] we consider a continuous, beam with a parabolic charge density, an elliptical transverse cross section, and zero initial emittance. We will suppose that as the beam travels over a short distance $z$, the space-charge impulse changes the transverse momentum of the particles, but the particle positions do not change appreciably; we assume that the parabolic density profile is constant. The model describes the initial stages of the charge redistribution process, and predicts how the emittance growth depends on the parameters. In $x-x^{\prime}$ space there are two effects, 1) filamentation that changes an initial straight line in phase space (representing the zero-emittance initial beam) into a curved line, and 2) an $x-y$ coupling through the space-charge term, which produces a spreading of the curved filament into a finite phase-space area in $x-x$ ' space, as shown in Fig. 1 .

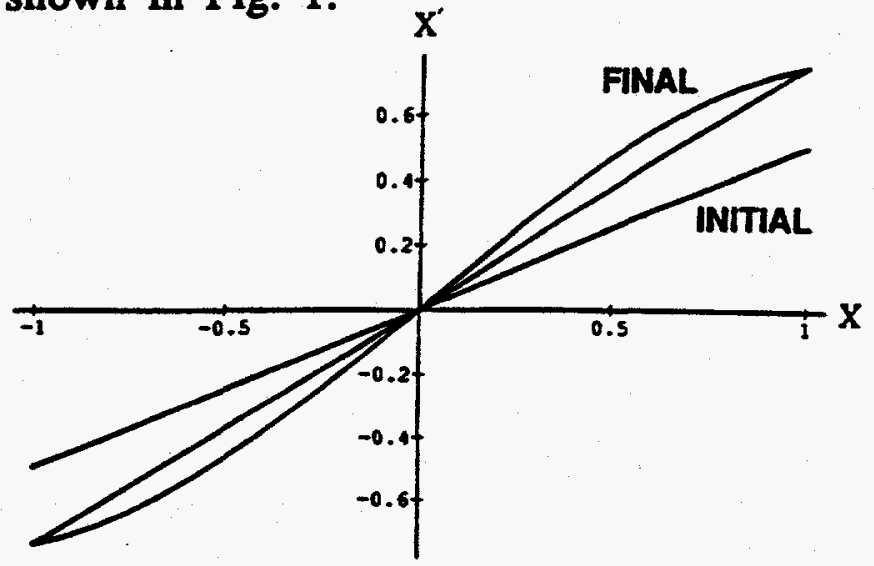

Figure 1. Effect from the model of the space-charge force on a parabolic-density beam with zero initial emittance. Initial and final phase-space distributions are shown. 
The rms emittance grows linearly with respect to $z$, and for a circular beam, we find

$$
\varepsilon_{\mathrm{x}}(z)=\frac{\mathrm{Kz}}{12 \sqrt{5}}
$$

where $\mathrm{K}$ is the generalized perveance. The space-chargeinduced emittance growth rate is independent of the rms beam size, which is the result of two compensating effects. As the beam size increases, the average space-charge force, and the transverse momentum impulses from space-charge decrease. But at fixed initial $\varepsilon x$, the smaller momentum impulses are distributed over a larger beam, and the total phase-space area increase is independent of the beam size.

To obtain a prediction for the final emittance growth, it is necessary to take into account the saturation of the emittance growth that usually occurs after the transverse movement of the particles is included, and the beam relaxes into a matched density configuration. This stage of the dynamics is not easily adapted to a detailed dynamic model. However, we can appeal to the results from the numerical simulations, which tell us that the emittance growth of an initially rms-matched beam saturates after the beam has propagated for one quarter plasma period $[6,21]$ The beam-plasma spatial period is related to the rms beam size a of the equivalent uniform beam by

$$
\lambda_{p}=\sqrt{2 \pi^{2} a^{2} / k} \text {. }
$$

Suppose we assume that the emittance grows linearly according to Eq.(8), while the beam propagates from $z=0$ to $\lambda_{p} / 4$. If the emittance growth rate is assumed to fall to zero at $z$ $=\lambda \mathrm{p} / 4$, as a result of the redistribution of the charges, we obtain for the final emittance

$$
\varepsilon_{x}\left(z=\lambda_{p} / 4\right)=\frac{K \lambda_{p}}{48 \sqrt{5}}=\frac{\pi}{48} \sqrt{\frac{2 K}{5}} a .
$$


The result of Eq.(10) gives the same functional dependences, and is only about $30 \%$ lower in magnitude than the result for a parabolic beam from nonlinear field energy theory, a theory that is in excellent agreement with the numerical simulations [6]. Eq.(10) shows the result that the saturated emittance growth increases with increasing beam size, which is a surprising result for an effect that is caused by space-charge forces, which decrease with increasing beam size. The result can be explained by the fact that larger beam size increases the plasma period, which means that for a large beam size, the emittance grows for a longer time before saturation, at a rate which is independent of the beam size. Furthermore, the appearance of the generalized perveance $K$ in these formulas shows that space-charge induced emittance growth is inherently a nonrelativistic effect, since $K \propto(\beta \gamma)^{-3}$. The model can also be adapted to describe the transverse emittance growth of a bunch with the shape of a right circular cylinder. For a bunched beam the emittance growth in the $x$ plane is affected by coupling to both the $y$ and the $z$ planes. Similar expressions are obtained in terms of a generalized perveance that has the form

$$
K=\frac{\mathrm{qI}_{\mathrm{ave}}}{4 \sqrt{3} \pi \varepsilon_{0} m \mathrm{~m}^{2} \beta^{2} \gamma^{3} \mathrm{bf}}
$$

where $I_{a v e}$ is the average current, $b$ is the rms bunch length, and $f$ is the bunch frequency. Eq.(11) predicts that to reduce $k$ and the emittance growth at fixed average current, one should choose a large bunch frequency to reduce the charge per bunch. This result implies that bunched-beam emittance growth is less for a higher frequency linac, and is a consequence of a smaller number of particles per bunch for a fixed average current at higher frequency.

\section{BEAM HALO}

The subject of beam halo has received much attention during the past several years, as is indicated by the sample given in references [22] to [29]. The phenomenon of beam halo has 
practical importance because beam loss can result, if the halo is intercepted by the apertures of accelerator structures. Even a beam loss rate as small as $1 \mathrm{nA} / \mathrm{m}$ at about $1 \mathrm{GeV}$, can cause significant radioactivation corresponding to a dose rate of approximately $20 \mathrm{mrem} / \mathrm{hr}$ at a distance of $30 \mathrm{~cm}$ from the structure at 1 hour after beam is turned off. There are many unanswered questions about the origin and properties of the halo. It is known that the halo particles are those that possess much higher than average energies of transverse motion. It is known from computer simulations that halos are produced from the space-charge-induced emittance growth processes, especially from the emittance growth that occurs in an initially rms-mismatched beam. This is not unexpected physically, because mismatched beams produce time-varying spacecharge forces from the oscillations of the core of the beam that can, under certain conditions, transfer energy to a small fraction of particles, such as those that are initially just outside the main core. A precise definition of the halo does not exist; a simple and useful definition is to define the halo to include all particles that are outside an ellipse with the same aspect ratio as the rms ellipse, and with an emittance of 4 times the rms emittance for a continuous beam, and 5 times the rms emittance for a bunched beam. The factors of 4 and 5 correspond to the total emittance of the equivalent uniform beams, uniform beams that have the same first and second moments as the real beam.

Emittance growth is often compared with the classical filamentation process, where in one-dimensional motion a continuous nonlinear force produces oscillation frequencies that depend on the particle amplitudes. This results in a curvature, when an initial phase-space ellipse becomes distorted into an s-shape, and eventually into a spiral structure in phase space. However, computer simulation studies show that after a few plasma periods, a round and initially rms-mismatched beam develops in $x-x^{\prime}$ phase space into an inner core and an outer halo, instead of the classical spiral. To investigate the connection between the classical filamentation and the halo, it is revealing to examine at the evolution of an initial zeroemittance, radially mismatched beam, where all particles 
undergo radial oscillations. The beam oscillate: in a azimuthally symmetric breathing, which is conveniently viewed in the radial $r-r^{\prime}$ plane. One finds that filamentation is observed in $r-r^{\prime}$ space, although the filamentary structure is more complicated than a simple spiral, because of the time dependence of the nonlinear space-charge force. The initial straight line in phase space rapidly evolves to a filament that is bent back and forth many times, forming dense inner core, and an outer spiral-like structure. The spiral-like filament that is observed in $r-r$ ' space projects into a diffuse halo in $x-x$ ' space, because of the coupling of the $x$ and $y$ motions. Thus, the formation of a core and halo is found to result from the fundamental filamentation process, coupled with the projection onto the Cartesian $x-x^{\prime}$ phase space. Similar spreading effects from coupling, as seen in the projected phase-space distribution, were described in the emittance-growth model of the previous section.

Recently, the particle-core model has been studied to provide a better understanding of the mechanism that transfers energy to particles, resulting in halo formation. In this model we consider a continuous beam in a uniform focusing channel. A round, continuous, uniform-density distribution is used as a model for the core. The core is mismatched, so the radius of the core will oscillate in a breathing mode with an azimuthally symmetric core displacement. The halo particles are represented by test particles, which oscillate through the core, influenced by linear external focusing fields, and the nonlinear space-charge fields of the core. The object of the model is to study the dynamics of the test particles. The model consists of the envelope equation, and the single particle equation of motion, which are solved simultaneously. In dimensionless form these equations may be written as

and

$$
\frac{d^{2} X}{d s^{2}}+X-\frac{\eta^{2}}{X^{3}}-\frac{I}{X}=0,
$$

$$
\frac{d^{2} x}{d s^{2}}+x-\left\{\begin{array}{cc}
I x / X^{2}, & x<X \\
1 / x, & x \geq X
\end{array},\right.
$$


where Eq.(12) describes $\mathrm{X}$, the ratio of the core radius to the matched beam radius, $s$ is the product of the axial distance times $k_{0}, \eta$ is the tune-depression ratio of the core, or $k / k_{0}$, where $k$ is the single particle transverse phase advance per unit length, $k_{0}$ is the same quantity without the space-charge force, and $I=1-\eta^{2}$. Eq.(13) is the equation of motion of a single particle, where the normalized displacement $x$ is defined as the ratio of the displacement to the matched $\mathrm{mms}$ radius of the core. We note that the two equations contain a single dimensionless parameter $\eta$. The motion of a particle depends on the normalized core radius $\mathrm{X}$, but the dynamics of the core is independent of the motion of the particle. This is a reasonable approximation, if the halo particles represent only a small fraction of the total beam.

The system is studied by systematically varying the initial state of the particle. A stroboscopic plot of the problem, obtained by taking snapshots of many independent particle trajectories, once per core-oscillation cycle at a specified phase of the core oscillation, reveals a separatrix defining three kinds of trajectories, 1) core dominated, 2) resonance dominated, and 3) focusing dominated. The plot is shown in Fig. 2. The coredominated trajectories are the innermost trajectories on the stroboscopic plot. The focusing-dominated trajectories are the outermost trajectories, which describe a peanut-like shape in the plot. The resonance-dominated trajectories are most strongly affected by a parametric resonance, which occurs when the particle frequency is approximately half the core frequency. This resonance is responsible for the growth of the amplitudes that can form a halo. Particles that are initially nearest the core, and within the resonance-dominated region, are driven by the resonance, and acquire large amplitudes. The growth of the amplitudes is self limiting, because of the nonlinear decrease of the space-charge force outside the core. The model predicts an important result, that a maximum amplitude exists, which is the largest displacement on the separatrix, corresponding to a stroboscopic phase when the core has the minimum size. Resonance-dominated particles will cycle out and back as a result of the resonance, but because of the amplitude-dependence of the frequency, a significant phase 
mixing occurs, and there will usually be some particles with amplitudes near the maximum value. It is found that the model results are insensitive to the details of the charge-distribution of the core. This has been determined by study of a model where the uniform core distribution is replaced with a Gaussian distribution. Although the Gaussian distribution is not generally an equilibrium distribution in a linear focusing channel, this replacement exercise is still valid for testing the sensitivity of the results to the assumed core distribution.

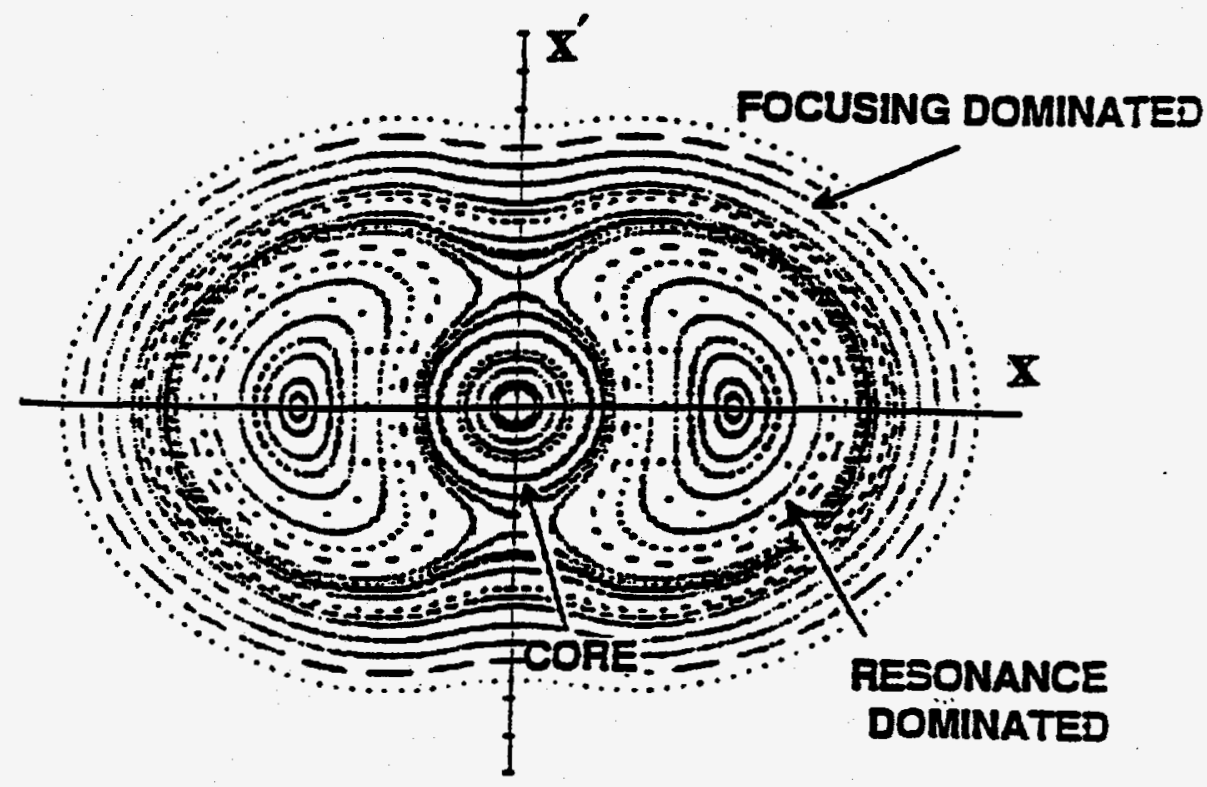

Figure 2. Stroboscopic plot obtained by taking snapshots of many independent particle trajectories, once per core-oscillation cycle at the phase of the core oscillation that gave minimum core radius. Initial coordinates were defined on the $x$ and $x^{\prime}$ axes. The three regions shown in the figure as described in the text.

Evidence for chaos, based on the breakup of the separatrix, is observed at low tune-depression ratios, when $\mathrm{k} / \mathrm{k}_{0}<0.4$. The growth time for halo in the resonance-dominated region can be estimated from the model, as the average time required for particles within the resonance-dominated region to go from the minimum to the maximum amplitude. It is found that this halo-growth time varies; the typical observed growth time is about 5 to 10 core cycles for space-charge dominated beams with $\mathrm{k} / \mathrm{kO}<0.5$, and is about 30 to 40 cycles for $\mathrm{k} / \mathrm{kO}=0.9$. 
estimated from the model, as the average time required for particles within the resonance-dominated region to go from the minimum to the maximum amplitude. It is found that this halo-growth time varies; the typical observed growth time is about 5 to 10 core cycles for space-charge dominated beams with $\mathrm{k} / \mathrm{k} 0<0.5$, and is about 30 to 40 cycles for $\mathrm{k} / \mathrm{kO}=0.9$.

There are many questions remaining about halos. These include: 1) Do other modes of a mismatched beam contribute significantly to halo formation besides the breathing mode? 2) When other modes are included, is there a maximum halo amplitude? 3) How are the predictions of the particle-core model affected by periodic focusing instead of the uniform focusing? 4) What new effects are introduced from longitudinally mismatched beams?

\section{EQUIPARTITONING}

A fundamental unanswered problem is that of predicting the evolution of a beam, whose initial state is given, when binary collisions, which would lead to thermal equilibrium and a Maxwell-Boltzmann distribution, are neglected. At. present, we ... are able to predict the evolution only by direct computer simulation. Nevertheless, it has been suggested that the concepts of statistical mechanics and thermodynamics may be useful for describing beams, even when binary collisions are unimportant.

One such question is that of energy equipartitioning of beams, caused by collective space-charge effects. The space-chargeinduced coupling between the motion in different planes provides a mechanism for energy transfer between planes. Does this lead to equipartitioning of the beam? To formulate the problem, suppose we consider the simple case of an rmsmatched beam with uniform focusing in all three planes. In general the beam is not in thermal equilibrium. Suppose we define a temperature for each plane, by transforming to the center-of-momentum frame of the beam or beam frame, and define temperatures in the beam frame according to 


$$
k_{B} T_{X} \equiv \frac{\overline{p_{b x}^{2}}}{m}, k_{B} T_{y} \equiv \frac{\overline{p_{b y}^{2}}}{m}, k_{B} T_{z} \equiv \frac{\overline{p_{b z}^{2}}}{m} .
$$

Equipartitioning may be defined as $k_{B} T_{x}=k_{B} T_{y}=k_{B} T_{z}$. By performing a Lorentz transformation back to the laboratory frame, and using the definition of rms normalized emittances, we can show that equipartitioning implies that

$$
\frac{\varepsilon_{n, x}^{2}}{x_{\text {rms }}^{2}}=\frac{\varepsilon_{n, y}^{2}}{Y_{\text {rms }}^{2}}=\frac{\varepsilon_{n z}^{2}}{\gamma^{2} z_{x m s}^{2}},
$$

If we consider the single particle equations of motion for an equivalent uniform beam, we can show that the rms beam sizes can be written as

$$
x_{r m s}^{2}=\frac{\varepsilon_{n, x}}{\beta \gamma k_{x}}, \quad y_{r m s}^{2}=\frac{\varepsilon_{n, y}}{\beta \gamma k_{y}}, \quad z_{r m s}^{2}=\frac{\varepsilon_{n z}}{\beta \gamma^{3} k_{z}},
$$

where $k_{x}, k_{y}$, and $k_{z}$ are the phase-advances per unit length of the equivalent uniform beam including space charge, and $g$ is the relativistic mass factor for the center of momentum. Substituting Eqs.(15) into (16), we obtain for a convenient expression of the equipartitioning condition

$$
\varepsilon_{x} k_{x}=\varepsilon_{y} k_{y}=\varepsilon_{z} k_{z}
$$

However, equipartitioning is by no means an established principal for collisionless beams. Earlier work by Hofmann [11] identified a coherent anisotropy instability, which affects nonequipartitioned beams. Several questions can be asked to address the significance of equipartitioning for space-charge dominate beams. Three such questions are presented by Hofmann at this conference. 1) How large an unbalance of Eq.(17) can exist through thermal anisotropy without exciting an instability that restores equipartitioning? 2) If an instability is induced by thermal anisotropy, will the beam evolve until Eq.(17) is established? 3) Will such a process produce beam halo, especially in the initially cold degree of freedom, which is 
warmed by the energy-transfer process? Hofmann's paper gives some answers to these questions for a 2-D beam. Additional work should be carried out to answer these and similar questions for 3-D bunched beams in linacs.

Other questions arise of a very practical nature for an accelerator designer? Assuming that exact equipartitioning will occur with undesirable consequences for possible production of beam halo and associated beam losses has led some to the suggestion that design procedures ought to give highest priority to injecting and maintaining equipartitioned beams in high intensity accelerators [30]. The question arises regarding what should be done, when numerical simulation studies show that thermal asymmetries develop in rms mismatched beams. Should the focusing system be tailored to maintain equipartitioning, even if this means weakening the focusing and increasing the rms beam size relative to the aperture radius, which can by itself lead to enhanced beam loss at high energies [31]. It may be that the optimum design approach depends on the detailed requirements of the application. Thus, the design approach for a proton linac that must inject beam into a storage ring may be different than for a linac that must deliver a high-power beam directly to a target. Nevertheless, it is clear that these are important questions that will need further study.

\section{CONCLUSION}

Significant progress has been made in understanding spacecharge-dominated beams, even since the 1991 University of Maryland High-Brightness Beam Workshop. Nevertheless there are still many unanswered questions about space-chargeinduced emittance growth, equipartitioning, and halo formation. Many of these questions are motivated by practical questions stimulated by the design of a new generation of high-current accelerators. 


\section{ACKNOWLEDGEMENTS}

The author thanks E. R. Gray and R. Garnett for some of the beam calculations discussed in the paper and for helpful discussions.

\section{REFERENCES}

1. J. D. Lawson, "The Physics of Charged Particle Beams," Clarendon Press, Oxford, Second Edition (1988) 151-155.

J. D. Lawson, "The Emittance Concept," AIP Conference Proceedings, (1991) 253.

2. C. Lejeune and J. Aubert, "Emittance and Brightness: Definitions and Measurements," Advanced Electronics and Electron Physics, Supplement 13A, Applied Charged Particle Optics, (1980) 184.

3. F. J. Sacherer, IEEE Transactions on Nuclear Science, 18 (3), 1105 (1971).

4. P. M. Lapostolle, IEEE Transactions on Nuclear Science, 18 (3), 1101 (1971).

5. J. D. Lawson, "The Physics of Charged Particle Beams,". Clarendon Press, Oxford, Second Edition , 190 (1988).

6. T. P. Wangler, K. R. Crandall, R. S. Mills, and M. Reiser, IEEE Transactions of Nuclear Science, 32 (5), 2196 (1985).

7. I. M. Kapchinskij and V. V. Vladimirskij, "Limitations of Proton Beam Current in a Strong Focusing Linear Accelerator Associated with Beam Space Charge," International Conference on High-Energy Accelerators and Instrumentation, (CERN, Geneva, 1959), p.274.

8. For a historical review of emittance growth for different accelerators, including a more complete set of references to 
early work, see T. P. Wangler, "Frontiers of Particle Beams: Intensity Limitations," Lecture notes in Physics 400, M. Dienes, M. Month, and S. Turner (Eds.), Springer-Verlag, Berlin, 542-561 (1992).

9. J. Struckmeier, J. Klabunde, and M. Reiser, Particle Accelerators 15, 47 (1984).

10. P. M. Lapostolle, Proceedings of the Proton Linear Accelerator Conference, Brookhaven National Laboratory Report BNL-50120 (1968).

11. I. Hoffman and I. Bozsik, Proceedings of the lLnear Accelerator Conference, Los Alamos National Laboratory document LA-9234-C (1982), p. 116.

12. R. A. Jameson, Proceedings of the Linear Accelerator Conference, Los Alamos National Laboratory document LA9234-C (1982), p.125.

13. M. Reiser, Proceedings of the Particle Accelerator Conference, IEEE Catalog No. 91CH3038-7 (1991), p. 2497.

14. A. Cucchetti, M. Reiser, and T. P. Wangler, Proceedings of the Particle Accelerator Conference, IEEE Catalog No. 91CH3038-7 (1991), p. 251.

15. R. L. Gluckstern, Proceedings of the Linear Accelerator Conference, Fermi National Accelerator Laboratory (1970), p.811.

16. I. Hoffman, L. J. Laslett, L. Smith, and I. Haber, Particle Accelerators 13, 145 (1983).

17. J. Stuckmeier and M. Reiser, Particle Accelerators 14, 227 (1984).

18. M. G. Tiefenback and D. Keefe, Transactions on Nuclear Science 32 (5), 2483 (1985). 
M. G. Tiefenback, "Space Charge Limits on the Transport of Ion Beams in a Long Alternating Gradient Systems," Ph. D. thesis, Lawrence Berkeley Laboratory, LBL-22465 (November 1986).

19. M. Reiser, "Theory and Design of Charged Particle Beams," John Wiley and Sons, Inc., New York, 1994, pp. 482-491.

M. Reiser, et. al., Physical Review Letters 61, 2933 (1988).

I. Haber, et. al., Physical Review A 44, 5194 (1991).

20. T. P. Wangler, P. Lapostolle, and A. Lombardi, Proceedings of the Particle Accelerator Conference, Washington, DC, (1993), pp. 3606-3608.

21. O. A. Anderson, Particle Accelerators 21, 197 (1987).

22. J. S. O'Connell, T. P. Wangler, R. S. Mills, and K. R. Crandall, Proceedings of the Particle Accelerator Conference, Washington, DC, (1993), p. 3657.

23. J. M. Lagniel, Nuclear Instrument and Methods in Physics Research A345, 46 (1994).

J. M. Lagniel, Nuclear Instrument Methods in Physics Research A345, 405 (1994).

24. R. L. Gluckstern, Physical Review Letters 73, 1247 (1994).

25. C. L. Bohn, Physical Review Letters 70, 932 (1993).

C. L. Bohn and J. R. Delayen, Physical Review Letters 50, 1516 (1994).

26. C. Chen and R. C. Davidson, Physical Review Letters 72, 2195 (1994).

Q. Qian and R. C. Davidson, Physics of Plasmas 2(7), 2674 (1995). 
Q. Qian, R. C. Davidson, and C. Chen, Physics Review E51(6), R5216 (1995).

27. C. Chen and R. A. Jameson, Phusical Review E52(3), 3074

28. S. Y. Lee and A. Riabko, Phusical Review E51, 1609
(1995).

A. Riabko, et. al., Phusical Review E51, 3529 (1995).

29. D.L. Bruhwiler, this conference.

30. M. Reiser, Proceedings of the Accelerator-Driven Transmutation Technologies Conference, Las Vegas, Nevada (1994) p. 364;

also see $M$. Reiser, et. al., this conference.

31. R. W. Garnett, et. al. "Linear Accelerator for Tritium Production", this conference.

\section{DISCLAIMER} United States Government nor any agency thereof, nor any of their Government. Neither the United States Government legal liability or responsiemployees, makes any warranty, express or implied, of any information, apparatus, product, or bility for the accuracy, completeness, or usefuiness of any information, apparatus, product, Referprocess disclosed, or represents that its use would not infringe price by trade name, trademark, ence herein to any specific commercial product, process, or service by trade narsement, recommanufacturer, or otherwise does not necessarily constinent or any agency thereof. The views mendation, or favoring by the United States Government or any agen theflect those of the and opinions of authors expressed herein do not

United States Government or any agency thereof. 